\title{
Danksagung
}

Prof. Dr. Florian Coulmas

Preisträger

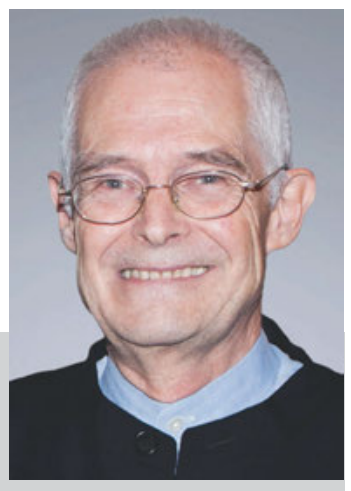

\section{Eine Frage der Zeit}

Magnifizenz, Spectabilis,

sehr geehrter Herr Kaiser,

meine sehr geehrten Damen und Herren,

lassen Sie mich damit beginnen, Ihnen dafür zu danken, dass Sie sich für diese Gelegenheit Zeit genommen haben. Sie hätten sie vielleicht viel besser verbringen können; die Zeit ist ja so kostbar, besonders in unserer Zeit. Das war nicht immer so, denn obwohl ein Lichtjahr für den Physiker ein Lichtjahr ist, hat der Sozialwissenschaftler große Mühe, die Zeit in objektive Begriffe zu fassen. Das liegt daran, dass wir als gesellschaftliche Wesen die Zeit auch erfahren und das heißt, auch subjektiv wahrnehmen, als Einzelne und als Gesellschaften.

Eine Stunde mit der Angebeteten verbracht, ist ein Augenschlag; eine Stunde im Stau, eine halbe Ewigkeit. Antonio Tabucchi, der begnadete italienische Schriftsteller, gab einem seiner letzten Bücher den Titel "Il tempo invecchia in fretta", was so viel bedeutet wie "Die Zeit altert in Eile". Wie kann die Zeit sich beeilen?

Ich gebe Ihnen ein Beispiel. Wir schreiben das Jahr 1873 und sind in Tokyo. Und auch dort schreibt man, noch etwas zaghaft zwar, aber 
doch fest entschlossen, das Jahr 1873. Das war etwas Neues, etwas ganz Neues. Bis dahin tat man das in Japan nicht, denn 1873 ist ja das Jahr des Herren, und dieser Herr war nicht Japans Herr. Der Herr der Zeit war immer der Tenno, der dafür eigens ein Amt unterhielt, das für Astronomie und Astrologie zuständig war. Wer Herr über die Zeit ist, hat das Sagen in praktisch allen Dingen. Das lässt man sich nicht so ohne Weiteres nehmen. Wie es trotzdem dazu kam, 1873 in Tokyo, und was es für Folgen hatte, das will ich Ihnen anhand einiger konkreter Beispiele erzählen.

Wir müssen etwas weiter zurückgehen, ins 16. Jahrhundert, als Japan zum ersten Mal mit dem Jahr des Herrn bzw. mit der christlichen Zeitrechnung in Berührung kam. Das war, als weltreisende Missionare nach Japan kamen, in ihrem Gepäck als Gastgeschenk, um dem Wort Gottes Überzeugungskraft und Nachdruck zu verleihen, die erste mechanische Uhr zusammen mit dem ersten Schießgewehr. Beide Geräte haben viel miteinander gemein. Aus vielen Einzelteilen zusammengesetzte Mechanismen, sind es Präzisionsinstrumente der Herrschaft, die die Welt verändert haben wie kaum eine andere Erfindung: der Donner der Kanonen und das Ticken der Uhr.

Wie Sie sicher wissen, ist die Uhrenindustrie heute nur noch teilweise in der Schweiz beheimatet. Sie wird von japanischen Firmen beherrscht, die Chronometer für die Olympischen Spiele und Armbanduhren für den Alltag herstellen. Das japanische Interesse an dieser Sparte der Ingenieurskunst reicht bis ins 16. Jahrhundert zurück. Anders als die Inder und die Chinesen, die von den europäischen Kolonisatoren ebenfalls Uhren als Gastgeschenke bekamen, sie aber als unnützen Tand ins Regal stellten, untersuchten die Japaner sogleich den Mechanismus, um davon möglicherweise etwas zu lernen. Unnütz war das Instrument schon, ebenso wie in Indien und China, denn in einer Agrargesellschaft lebt man nicht nach der Uhr. Was sollte man also mit diesem Wunderwerk der Technik? 
Die Antwort, die man auf diese Frage in Japan fand, resultierte in einem der merkwürdigsten Objekte der Technikgeschichte, der Wadokei oder, japanischen Uhr'.

Zunächst war das ein Prestigeobjekt, das sich nur Feudalherren leisten konnten, ein Zierstück. Aber sie sollte auch eine Funktion haben, die der Zeitmessung bzw. der Zeiteinteilung. Eine solche hatte man auch im vormodernen Japan. Der Tag war in zwölf koku oder Stunden unterteilt, sechs am Tage und sechs in der Nacht. Hier von Stunden zu sprechen, ist freilich irreführend, denn die koku waren in ihrer Dauer nicht bestimmt. Sie waren keine willkürliche Einheit, sondern Teil des jahreszeitlichen Rhythmus, der das Leben bestimmte.

Nur zweimal im Jahr waren die Tagstunden und die Nachtstunden gleich lang, nämlich zu Frühlingsanfang am 21. März und zu Herbstanfang am 22. September, den beiden sprechend sogenannten Tag-und-Nacht-Gleichen, die noch heute in Japan Feiertage sind. Im Sommerhalbjahr, wenn es lange hell ist, sind die Tagstunden lang, im Winter, wenn es lange dunkel ist, die Nachstunden.

Die zwölf koku entsprachen den zwölf Tierkreiszeichen des traditionellen Kalenders. Die ließen sich mühelos auf einem Zifferblatt abbilden. Ihre variable Länge war jedoch eine Herausforderung für die mechanische Uhr, die die Missionare mitgebracht hatten, denn die zeigte, wenn sie gut war, in ihrer unendlichen Langeweile, eine Stunde heute immer genauso lang an wie gestern und morgen und gleichviel, ob es Tag oder Nacht war. Ein solches Gerät war offensichtlich unbrauchbar, würde es doch nur zweimal im Jahr richtig gehen.

Aber so leicht geben japanische Bastler, Mechaniker, Ingenieure nicht auf. Konstruktionszeichnungen von Hosokawa Hanzo, auch als Bastel-Hanzo bekannt, lassen die Lösung des Problems deutlich erkennen. Die japanische Uhr hat zwei Unruhen, eine für tags und eine für nachts, jede mit einem Hebel versehen, um die Zeitmessung anzupassen. Die variable Stunde, deren Länge sich mit der Jahreszeit ändert, konterkariert den eigentlichen Zweck der mechanischen Uhr. 
Eine Uhr, der man zweimal am Tag sagen muss, wie spät es ist, das ist offenkundig eine Absurdität, jedenfalls aus Sicht derer, die es gewohnt sind, nach der Uhrzeit zu leben. Die Wadokei ist das Sinnbild, die Verkörperung des krampfhaften Versuchs, Unvereinbares miteinander zu vereinbaren und Gewohnheiten der vormodernen Lebensführung in die Moderne hinüberzuretten. Die Ereigniszeit begleitet das stark von den Jahreszeiten geprägte Leben agrarischer Gesellschaften. Die Uhrzeit wird erst mit der Synchronisierung des Lebens in der Industriegesellschaft relevant. Der Ereigniszeit in der Moderne einen Platz zu erhalten, war jedoch ein vergebliches Unterfangen, wie die Menschen schnell merkten, als das Jahr des Herren in Japan dann doch Einzug hielt, im 6. Jahr der Meiji-Ära, eben 1873.

Einer, der besser als die meisten seiner Zeitgenossen verstand, was die Stunde geschlagen hatte und dass die Kalenderreform mehr als nur symbolische Bedeutung hatte, war Fukuzawa Yukichi. Noch heute als einer der wichtigsten Aufklärer verehrt, ziert sein Konterfei sehr passend die 10.000-Yen-Note. Passend, weil er begriff, dass Modernisierung bedeutete, alles zu kommerzialisieren, auch und insbesondere die Zeit. Er begann damit, seinen Landsleuten zu erklären, wie man die neue Uhr lesen musste. Die kleine Broschüre, mit der er das tat, wurde zu einem Bestseller.

Andere folgten Fukuzawas Beispiel, und bald hatte jeder irgendein Heftchen, das das neue Zeitregime erklärte. Da in Japan die Bereitschaft zu lernen ebenso groß ist wie die, sich in Unvermeidliches zu fügen, setzte sich das neue Zeitregime schnell durch, obschon die Erinnerung an das alte, hergebrachte lebendig gehalten wurde. Im alltäglichen Leben machte es sich bemerkbar, indem immer mehr Menschen ihr Leben dem neuen Takt anpassen mussten und schon bald jeder Haushalt eine Uhr haben musste.

Als dann ein rundes Jahrzehnt, nachdem dieser Holzschnitt gedruckt wurde, im Jahre Meiji 22, 1889 die kaiserliche Verfassung verkündet wurde, war das neue Zeitregime fest etabliert. Bei diesem fei- 
erlichen Anlass wird der Meiji-Tenno, der dem Volk die Verfassung schenkte, im Thronsaal nur überragt von der westlichen Uhr.

Die Nähe der Uhr zur Schusswaffe kam dann auch bald zum Tragen, als die erste in Japan hergestellte Taschenuhr im Russisch-Japanischen Krieg 1904/05 eingesetzt wurde, um Angriffe zu synchronisieren und dann folgerichtig zur Tapferkeitsauszeichnung der Kaiserlichen Armee avancierte.

Der Hersteller war die Firma Seiko, deren Name Feinheit oder Erfolg bedeuten kann. Gründer der Firma war Hattori Kintaro, der Goldjunge - so sein Name wörtlich. ,Leben ist Arbeit' war die Maxime dieses Gründervaters, aus dessen Ein-Mann-Betrieb eine Weltmarke wurde, die heute jeder kennt und deren Haupthaus mit seinem Uhrturm in Ginza ein Wahrzeichen der Stadt Tokyo ist.

Der Übergang vom vormodernen zum modernen Zeitregime wurde sehr schnell vollzogen, Zeitdisziplin zu einer der hervorstechenden Tugenden Japans. Jedes Kinderzimmer wurde mit einer Uhr bestückt, damit der künstliche Rhythmus zur zweiten Natur wurde. Just in time wurde nach der Erfindung des Fließbands zu einer der wichtigsten Neuerungen der industriellen Produktionsweise. Und die Pünktlichkeit der öffentlichen Verkehrsmittel wurde zum Neid aller anderen Industrieländer, insbesondere Deutschlands, wo sich ältere Mitbürger noch heute daran erinnern, dass es beinah sprichwörtlich hieß, ,pünktlich wie die Eisenbahn'. In Japan gilt das uneingeschränkt. Wer sein Zuspätkommen am Arbeitsplatz oder in der Schule mit einer Verspätung des Zuges entschuldigen will, ist unglaubwürdig. Niemand glaubt ihm, da jeder weiß, dass sich Verspätungen aufs Jahr gerechnet in wenigen Minuten bemessen. Die Verkehrsbetriebe stellen deshalb, wenn es denn tatsächlich einmal zu einer Verspätung kommt, Verspätungszertifikate aus, die den Passagieren an der Ausgangssperre überreicht werden.

Man hat keine Zeit zu verlieren. Tokyo ist heute die schnelllebigste Stadt der Welt. Danach befragt, was für sie die wichtigste japanische Erfindung des 20. Jahrhunderts sei, antwortete eine Mehrheit 
der Tokyoter: Cupnoodles, das Fertiggericht der Instant-Nudeln, das in drei Minuten bereitet und in fünf Minuten verzehrt ist. Nicht überraschend ist es da, dass dieser Erfindung ein eigenes Museum gewidmet ist. Es steht, wenn wir an die Bedeutung dieser Stadt für die Modernisierung Japans denken, durchaus passend in Yokohama.

Ein Zeitregime zu ändern, ist sehr schwierig, so schwierig fast wie ein Sprachregime und ein religiöses Regime, denn wie jene ist es nicht nur mit der Kultur verbunden, sondern ein zentraler Bestandteil derselben. Es ist kein Zufall, dass in Japan, wo der Gregorianische Kalender und die darauf beruhende Zeitmessung relativ jung sind, wiederholte Versuche unternommen wurden, das Zeitregime zu reformieren, denn die sexagesimale Zeitrechnung ist zweifellos ein Anachronismus. Aber weder die metrische Uhr noch die Uhr für den 28-Stunden-Tag der 6-Tage-Woche, Montu, setzte sich durch. Zu eingeschliffen und kulturell verankert sind die sexagesimale Zeitrechnung und die 7-Tage-Woche. Also ist man einstweilen auch in Japan dabei geblieben.

Naturgegeben ist das freilich nicht. Die Wadokei, die japanische Uhr, ist auf dem Abfallhaufen der Geschichte gelandet, aber die Sehnsucht nach der guten alten Zeit ist noch immer augenfällig. Nach einem 6-Tage-Zyklus aus vormoderner Zeit bestimmen noch immer die meisten Japanerinnen und Japaner den Tag ihrer Hochzeit. Die meisten Tageszeitungen schreiben das moderne und das traditionelle Datum auf ihre erste Seite. Auf vielen formellen Einladungen steht die Jahreszahl der gegenwärtigen Ära. Schon kann man in Restaurants Reservierungen machen, um den Jahreswechsel Heisei 28/29 zu feiern. Und überhaupt nennt man bei traditionellen Feiertagen gern die Jahreszahl der traditionellen Zählung, obwohl das rein dekorativen Charakter hat, denn die Tage sind dem Gregorianischen Kalender bzw. dem Kalender der internationalen Wertpapierbörsen angepasst.

Aber die Zeiten ändern sich. Heute erleben die Japaner mehr Festtage als früher, doppelt so viele wie in der Meiji-Zeit; schließlich hat sich im Laufe des kurzen 20. Jahrhunderts auch ihre Lebenserwar- 
tung verdoppelt. Haben die Japaner deshalb mehr Zeit? Wer würde das im Zeitalter der "Tyrannei des Augenblicks“ (Thomas Hyland Eriksen) zu behaupten wagen?

Ich hoffe, meine sehr verehrten Damen und Herren, ich habe Ihnen hiermit einen kleinen Einblick in die Ethno-Chronologie gegeben, einen Wissenschaftszweig, der sich mit Fragen der Zeit in verschiedenen Gesellschaften und Kulturen beschäftigt: Wie viel Zeit verbringen die Menschen am Arbeitsplatz, im Park, in der Schule, in der U-Bahn, im Theater, im Gefängnis, am Esstisch, im Gespräch mit Freunden, im Gebet, im Bett, an ihrem Smartphone oder damit, einem Vortrag am Mittwochabend zuzuhören? Wenn Sie all die Antworten kennen, dann wissen Sie viel über eine Gesellschaft. Und vielleicht können Sie jetzt etwas besser nachvollziehen, warum die Uhren in Japan anders gehen - wie man so sagt.

Ich komme zum Schluss - eine Frage der Zeit. Eine Frage der Zeit ist es auch, dass man geehrt wird. Damit will ich nicht sagen, dass es an der Zeit war, keineswegs, sondern vielmehr, dass es auch Freuden gibt, wenn die Jugend vorbei ist. Ich fühle mich geehrt und freue mich ungemein. Ich danke der Stiftung und dem Preiskomitee von Herzen. Ich danke denjenigen, die auf den kuriosen Gedanken kamen, mich für diesen großartigen Preis zu nominieren - und ich danke Ihnen allen für Ihre Zeit.

\section{Prof. Dr. Florian Coulmas (geb. 1949)}

Lehrte von 1987 bis 1999 an der Chūō University in Tokyo, Sociology of Language.

Seit 1999 ist er Professor für Sprache und Kultur des modernen Japan am IN-EAST Institut für Ostasienstudien der Universität Duisburg-Essen, seit 2015 ist er an diesem Institut Senior-Professor.

Von 2004 bis 2014 war er Direktor des Deutschen Instituts für Japanstudien in Tokyo. 
